\title{
Inervación del Músculo Extensor Radial Largo del Carpo en Individuos Brasileños: Biometría de su Origen y Distribución
}

\author{
Innervation of the Extensor Carpi Radialis Longus muscle in \\ Brazilian individuals: Biometry of its Origin and Distribution
}

Pérez Mérida, L. ${ }^{1,2,3}$; Sousa Rodrigues, C.F. ${ }^{4}$ \& Olave, E. ${ }^{5}$

PÉREZ MÉRIDA, L.; SOUSA RODRIGUES, C.F. \& OLAVE, E.Inervación del músculo extensor radial largo del carpo en individuos brasileños: Biometría de su origen y distribución. Int. J. Morphol., 37(2):423-427, 2019.

RESUMEN: El músculo extensor radial largo del carpo (MERLC) es un músculo localizado en el compartimiento posterior (extensor-supinador) del antebrazo y tiene gran importancia en el cierre del puño. Hay pocos estudios biométricos con respecto al punto de origen de sus ramos de inervación, así como sobre la distribución de los mismos. Basado en lo anterior, se estudiaron 30 miembros superiores, formolizados, de individuos adultos Brasileños, de la Facultad de Medicina de la Universidad Estadual de Ciencias da Saúde de Alagoas, Maceió, Brasil. Luego de localizar el nervio, se midió la distancia entre el origen del ramo primario y el de los puntos motores respecto a la línea biepicondílea (LBE), los cuales fueron visualizados y disecados utilizando una lupa. El nervio en cuestión, se observó a nivel del brazo o proximal a LBE en 28 casos (93\%) y los 2 restantes a nivel de esta línea (7 \%). Los ramos para el ERLC se originaron a partir del nervio radial, observando un ramo primario en 20 miembros ( $80 \%)$, y en los restantes 10 (20\%) se observaron 2 ramos primarios, promediando 1,3 ramos (DS 0,5). El origen más proximal del primer ramo primario (RP) independiente de que si existían 1 o 2 fue en promedio $3 \mathrm{~cm}$ (DS 1,0) proximal a LBE. El PM más distal, se ubicó distal a LBE en 24 casos con un promedio de 1,9 cm (DS 1,0); localización a nivel de LBE en 3 casos. Sólo en 3 casos el PM más distal se encontró proximal a LBE, en un promedio de $0,8 \mathrm{~cm}$ (DS 0,5). La distribución de puntos motores fue variable, ya que muchas veces los RP se bifurcaban en ramos secundarios y éstos, a su vez se dividían hasta 6 veces en ramos terciarios que penetraban en el músculo. El conocimiento biométrico del origen del nervio del MERLC, así como su distribución, es un aporte importante al área anátomo-quirúrgica, así como, su utilización en bloqueos nerviosos, transferencias nerviosas y zonas de ubicación de electrodos con propósitos de estimulación eléctrica en pacientes que necesiten rehabilitar la acción de musculatura extensora radial lesionada.

PALABRAS CLAVE: Anatomía; Nervio radial; Músculo extensor radial largo del carpo; Puntos motores.

\section{INTRODUCCIÓN}

El músculo extensor radial largo del carpo (MERLC) es un músculo fusiforme que se solapa parcialmente con el músculo braquiorradial y que se encuentra localizado en el compartimiento posterior (extensorsupinador) del antebrazo y está inervado por ramos del nervio radial (NR) (Moore et al., 2013). Son pocos los estudios que muestren un análisis de las distancias de origen y la distribución nerviosa en este músculo.

El origen del nervio para el MERLC, según Hovelaque (1927), se ubica 1 o $2 \mathrm{~cm}$ distal al nervio para el Músculo Braquiorradial, el cual posteriormente se profundiza y avanza verticalmente hasta llegar al vientre mus- cular. Es aquí en donde penetra generalmente a nivel del epicóndilo lateral (EL).

Para Branovacki et al. (1998) este nervio podía originarse proximal al origen del nervio para el músculo braquiorradial en $10 \%$ o al mismo nivel de éste en $20 \%$, mientras que en el $70 \%$, éste se originaba a un nivel distal al nervio de referencia en una muestra de 60 especímenes.

Por otro lado, el nervio para el MERLC ha sido utilizado como nervio receptor en las transferencias nerviosas para restablecer la extension de muñeca, a partír del nervio para el músculo pronador redondo (García-

\footnotetext{
${ }^{1}$ Departamento de Anatomia y Medicina Legal, Universidad de Chile, Santiago, Chile.

${ }^{2}$ Departamento de Anatomia, Universidad Central de Chile, Santiago, Chile.

${ }^{3}$ Departamento de Anatomia, Universidad Autónoma de Chile, Santiago, Chile.

${ }^{4}$ Universidade Estadual de Ciencias da Saude de Alagoas, Maceió, Brasil.

${ }^{5}$ Facultad de Medicina, Universidad de La Frontera, Chile.
} 
López et al., 2014) o a partir del nervio musculocutáneo, específicamente el ramo para el músculo braquial en un caso de tetraplejia posterior a lesión medular C5 (Fridén \& Gohritz, 2012). Pet et al. (2011) utilizaron este nervio como donante para la recuperación de la extensión del codo posterior a lesión del plexo braquial, transfiriéndolo hacia ramos del tríceps braquial, obteniendo muy buenos resultados.

El objetivo de este estudio fue identificar las distancias de origen de este nervio con respecto a la línea biepicondilea (LBE) y analizar su distribución nerviosa a nivel del músculo extensor radial largo del carpo.

\section{MATERIAL Y MÉTODO}

Se utilizaron 30 miembros superiores de individuos adultos brasileños, de la Facultad de Medicina de la Universidad Estadual de Ciencias da Saúde de Alagoas, Maceió, Brasil. Las piezas cadavéricas se encontraban fijadas en solución de formaldehído al $10 \%$, de los cuales 15 miembros eran derechos y 15 izquierdos. La disección de la región cubital se realizó con los miembros superiores en posición anatómica, en extensión y con material quirúrgico adecuado.

Luego de ubicar el nervio a nivel del margen medial del músculo extensor radial corto del carpo, se procedió a medir el origen y distancia de los puntos motores respecto a LBE, conformada por las márgenes más prominentes, lateral y medial, de ambos epicóndilos. A partir de este límite se midió con huincha métrica, el origen del nervio a nivel del o los ramos terminales del NR, así como los puntos motores penetrando a nivel del vientre muscular, los cuales debían ser visualizados y disecados a través de una lupa. La anatomía de la división de este nervio se padronizó en relación a la división de él o los ramos principales.

\section{RESULTADOS}

Ramos Primarios. Con respecto al origen de este nervio, se ubicó mayoritariamente a nivel de brazo o proximal a LBE en 28 casos ( $93 \%$ ), mientras que en los 2 restantes se ubicó a nivel de esta línea (7\%). Todos los ramos para el ERLC se originaron a partir del nervio radial, observando un ramo primario en 20 miembros $(80 \%)$, mientras que en 10 casos $(20 \%)$ se observaron 2 ramos primarios, promediando 1,3 ramos (DS 0,5) (Fig. 1).

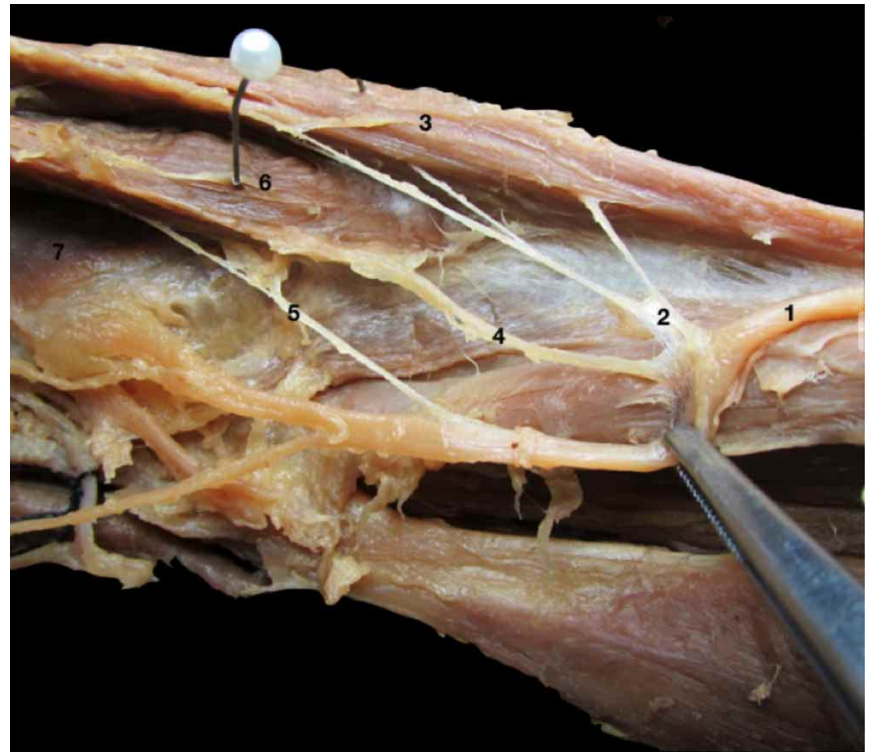

Fig 1. Dos ramos primarios a partir del nervio radial: 1: Nervio radial, 2: Ramo para el braquiorradial, 3: Músculo braquiorradial, 4: Primer ramo primario para MERLC, 5: Segundo ramo primario para MERLC, 6: Músculo extensor radial largo del carpo, 7: Músculo extensor radial corto del carpo.

Biometría del Origen del Ramo(s) Primario(s): En promedio el origen más proximal del primer ramo primario (RP) independiente de que si existían 1 o 2 fue de $3 \mathrm{~cm}$ (DS 1,0$)$ proximal a LBE.

Cuando solo existía un RP el promedio de origen alcanzó 2,7 cm (DS 0,96) proximal a LBE, cuando existían 2 RP la distancia de origen promedio fue de 3,15 y $1,85 \mathrm{~cm}$ proximal a $\mathrm{LBE}$, respectivamente. Solo en dos casos encontramos el origen de este nervio al mismo nivel de LBE.

Puntos Motores: En promedio el PM más proximal se localizó a $1,3 \mathrm{~cm}$ (DS 0,8$)$ proximal a LBE en 10 casos y a nivel de ésta en 10 casos. Cuando el PM más proximal se ubicó distal a LBE, éste estuvo en promedio a 1,3 cm (DS $1,0)$ en 10 casos. Para el PM más distal, éste se ubicó distal a LBE en 24 casos con un promedio de $1,9 \mathrm{~cm}$ (DS 1,0) distal; localización a nivel de LBE en 3 casos. Sólo en 3 casos el PM más distal se encontró proximal a LBE, en un promedio de $0,8 \mathrm{~cm}$ (DS 0,5) proximal. Por lo tanto, el rango de distancia en donde se encuentran los PM del nervio para el MERLC se ubican a $1,3 \mathrm{~cm}$ proximal y 1,7 $\mathrm{cm}$ distal a LBE (Intervalo de $3 \mathrm{~cm}$ ).

La distribución de puntos motores fue variable, ya que muchas veces los ramos primarios se bifurcaban en ramos secundarios y éstos a su vez también podían bifurcarse 
y hasta dividirse 6 veces en filetes nerviosos o ramos terciarios que penetraban a nivel muscular. De los 20 ramos primarios, solo 8 ingresaron directamente a nivel muscular sin ramificaciones, generando $1 \mathrm{PM}$, mientras que en 12 casos este ramo primario se bifurcó en 9 y trifurcó en 3 generando 2 y 3 PM respectivamente. Es importante mencionar que cuando existían ramos bifurcados uno de los RS se dividía de 3 a 6 PM que penetraban a nivel muscular (ramillete nervioso) abarcando un área de $1 \mathrm{~cm}$ aproximadamente (Fig. 2).

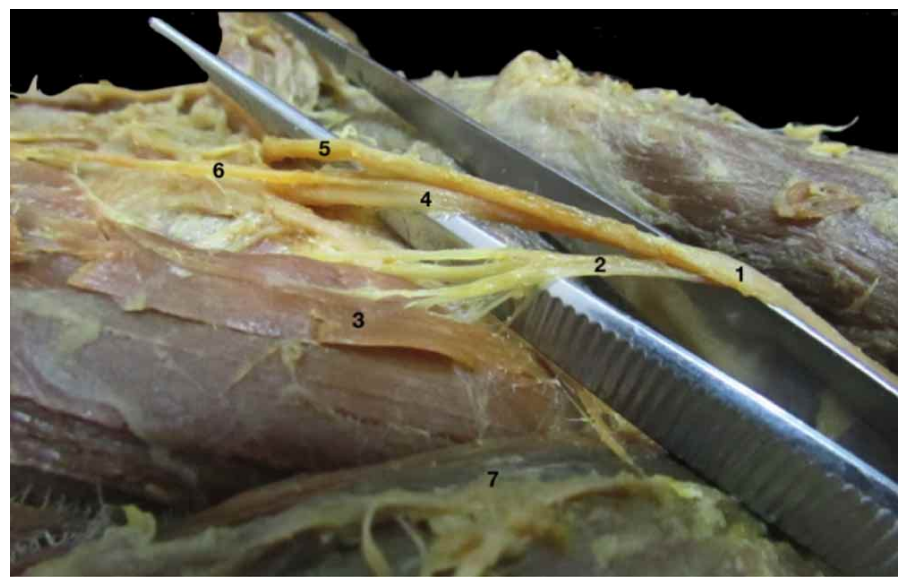

Fig 2. Ramillete nervioso localizado en MERLC. 1: Nervio radial, 2 Ramo primario para MERLC, 3: Músculo extensor radial largo del carpo, 4: Ramo profundo del nervio radial, 5: Ramo superficial del nervio radial, 6: Ramo para el músculo extensor radial corto del carpo.

Cuando se observaron 2 ramos primarios, en 2 casos ambos ramos primarios penetraron directamente el vientre muscular, generando $2 \mathrm{PM}$.

En 5 ocasiones uno de los RS penetró ramificado en 2, 3 o más filetes nerviosos (ramillete nervioso) mientras que el otro RS lo hizo directamente, generando hasta 6 PM, mientras que en 3 especímenes, los ramos secundarios se bifurcaban respectivamente, para penetrar en el músculo, generando 4 PM.

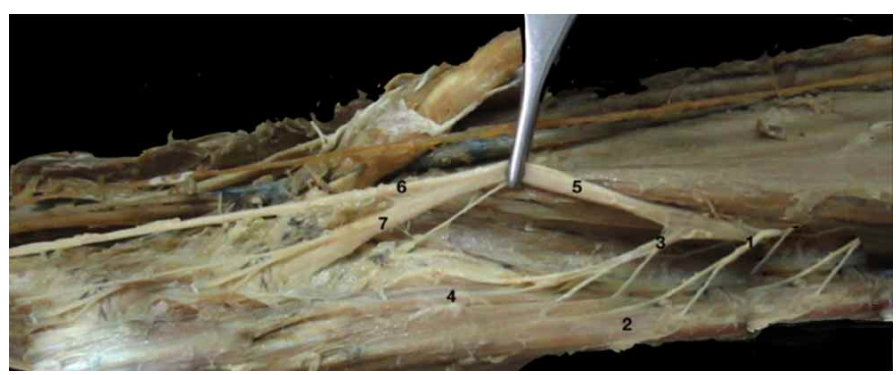

Fig 3. Tronco común para músculo braquiorradial y MERLC. 1: Ramo para el músculo braquiorradial, 2: Músculo braquiorradial, 3: Tronco común para el músculo braquiorradial y MERLC, 4: Músculo extensor radial largo del carpo, 5: Nervio radial, 6: Ramo superficial del NR, 7: Ramo profundo del NR.
Distribución Nerviosa Compartida. En al menos 5 miembros (17\%) la inervación para el MERLC brindó filetes nerviosos para el músculo braquiorradial (Fig. 3), mientras que en 1 caso (3\%) la inervación del MERLC se compartió con un filete nervioso que se dirigía hacia el MERCC.

\section{DISCUSIÓN}

Con respecto a su localización, en nuestro estudio el origen del nervio para el MERLC se ubicó en su mayoría distal al nervio para el músculo braquiorradial. Branovacki et al. localizó en $70 \%$ la rama del ERLC distal a la rama del músculo braquiorradial.

Hovelacque (1927) menciona que una estadística realizada por Linell, en una muestra de 22 casos, se observó que la penetración a nivel muscular es 11 veces en el brazo, 7 veces en el antebrazo y 4 veces en el vértice del epicóndilo lateral. En un caso anormal, el nervio surgió del nervio interóseo posterior y alcanzó el músculo $5 \mathrm{~cm}$ distal al epicóndilo. Estos estudios incluyeron un origen distal al EL que no fueron observados en esta investigación, así como tampoco un origen a partir del ramo profundo del nervio radial (nervio interóseo posterior).

Por otro lado, Sunderland (1946) observaron en todas sus muestras al primer ramo primario originándose proximal al epicóndilo. Detalló un suministro suplementario desde el nervio radial en 8 especímenes, desde el sitio de su división en 3, desde el ramo superficial en 1 , y desde el ramo profundo (nervio interóseo posterior) en 1. Las distancias de origen más alto y bajo del ramo primario se ubicaron a 5,6 y $0,3 \mathrm{~cm}$ en relación al epicóndilo, respectivamente. El ramo más distal, independientemente del número presente, dejó al nervio radial a 3 cm por distal al epicóndilo lateral. Konjengbam \& Elangbam (2004) en su estudio de atrapamiento del nervio radial, también ubicó un ramo para el MERLC, originándose a partir del ramo profundo del nervio radial.

En el estudio, el origen más alto del primer RP fue de 4,5 cm (1 caso) y el más bajo a nivel de la LBE (2 casos).

Con respecto al número de ramos primarios, Caetano et al. (2018) encontraron 1 a 2 ramos para 
el MERLC, en su muestra de 30 especímenes. Bertone et al. (2008) en su estudio del nervio para el MERLC encontró 1 ramo primario en $16(53,3 \%), 2$ ramos primarios en $5(16,7 \%)$ y a partir de un tronco común en 9 $(30 \%)$, a partir de su muestra de 30 miembros. En nuestro estudio observamos 6 casos de inervación compartida a partir de un tronco nervioso, 5 casos un tronco común para el músculo braquiorradial y 1 caso con distribución para el músculo extensor radial corto del carpo.

Branovacki et al., Safwat \& Abdel-Meguid (2007) y Ravichandiran et al. (2011) encontraron sólo un ramo primario en sus muestras, mientras que Segal et al. (1991) encontraron $2 \mathrm{RP}$ y Chen et al. (2009) reportaron 1,8 \pm 0,7 en promedio para los RP. En nuestro estudio se observó 1 RP en 20 casos (80 \%) y 2 RP en 10 (20\%), obteniendo un promedio de 1,3 (DS 0,5), aproximándose bastante a los estudios realizados por Liu et al. (1997) en cuyo estudio encontraron 1,2 $\pm 0,42$. De igual manera, Kerver et al. (2013) y Caetano et al. ubicaron 1 o 2 Ramos Primarios en sus estudios anatómicos.

Para los puntos motores la estadística es variable, en nuestro estudio observamos desde 1 hasta 6 PM, (Promedio 2,7 PM, DS 1,5) independiente de la cantidad de ramos primarios. Además 2,3 (DS 1,6) y 3,4 (DS 1,0) cuando se ubicó 1 y 2 RP respectivamente. Chen et al. registraron 2,3 $\pm 0,9 \mathrm{PM}$ y Kerver et al. ubicaron como promedio 2,5 PM (rango de 2 a 4). Nuestro estudio con 2,7 PM en promedio es concordante con estos últimos autores pero difiere a los observados por Liu et al., quienes registraron 3,3 $\pm 0,82$ y a los de Abrams et al. (1997) que informaron de 3,8 PM. Por otro lado, Kerver et al. mencionaron que el área de inervación para el músculo ERLC es 5 veces menor, en comparación con el músculo ERCC.

Como se puede apreciar, tanto la cantidad de ramos primarios como de puntos motores a nivel muscular es variable. Las distancias tanto de su origen como distribución son un importante hallazgo para el conocimiento anatómico, así como también para intervenciones quirúrgicas cercanas a su ubicación, bloqueos nerviosos, transferencias nerviosas y zonas de ubicación de electrodos para estimulación eléctrica en pacientes que necesiten rehabilitar la acción de la musculatura extensora radial.

PÉREZ MÉRIDA, L.; SOUSA RODRIGUES, C.F. \& OLAVE, E. Innervation of the extensor carpi radialis longus muscle in Brazilian individuals: Biometry of its origin and distribution. Int. J. Morphol., 37(2):423-427, 2019.
SUMMARY: The extensor carpi radialis longus muscle (ECRLm) is located in the posterior compartment (extensorsupinator) of the forearm and has great importance in the closure of the hand. There are few biometric studies with respect to the point of origin of their branches of innervation, as well as on the distribution of them. For this study, 30 upper limbs, formalized, of Brazilian adult individuals were used, from the Faculty of Medicine of the Universidad Estadual de Ciencias da Saúde de Alagoas, Maceió, Brazil. After locating the nerve, we measured the distance between the origin of the primary branch and that of the motor points with respect to biepicondilar line (BEl), which were visualized and dissected using a magnifying glass. The nerve in question was observed at the level of the arm or proximal to $\mathrm{BEl}$ in 28 cases $(93 \%)$ and the remaining 2 at the level of this line $(7 \%)$. The branches for the ECRLm originated from the radial nerve, observing a primary branch in 20 limbs (80\%), and in the remaining 10 (20\%) two primary branches were observed, averaging 1.3 branches (SD 0.5 ). The most proximal origin of the first primary branch $(\mathrm{PB})$ independent of whether there was 1 or 2 was on average $3 \mathrm{~cm}$ (SD 1.0) proximal to BEl. The most distal MP was distal to $\mathrm{BEl}$ in 24 cases with an average of $1.9 \mathrm{~cm}$ (SD 1.0); location at the BEl level in 3 cases. Only in 3 cases was the most distal MP found proximal to BEl, an average of $0.8 \mathrm{~cm}$ (SD 0.5). The distribution of motor points was variable, since many times the PB bifurcated into secondary branches and these, in turn, could divide up to 6 times in tertiary branches that penetrated in the muscle. The biometric knowledge of the origin of the nerve of the ECRLm, as well as its distribution, is an important contribution to the anatomo-surgical area, as well as its use in nerve blocks, nerve transfers and electrode placement areas for purposes of electrical stimulation in patients they need to rehabilitate the action of injured radial extensor musculature.

KEY WORDS: Anatomy; Radial nerve; Extensor carpi radialis longus muscle; Motor points.

\section{REFERENCIAS BIBLIOGRÁFICAS}

Abrams, R. A.; Ziets, R. J.; Lieber, R. L. \& Botte, M. J. Anatomy of the radial nerve motor branches in the forearm Anatomy of the radial nerve motor branches in the forearm. J. Hand Surg., 22(2):232-7, 1997.

Bertone, V. H.; Ottone, N. E.; Lo Tártaro, M. A.; García de Quirós, N.; Arrotea, A.; Domínguez, M.; Finkelstein, D.; López Bonardi, P.; Shinzato, S. \& Medan, C. Anatomical and Clinical considerations about the radial nerve in the elbow. Int. J. Morphol., 26(2):437-44, 2008.

Branovacki, G.; Hanson, M.; Cash, R. \& Gonzalez, M. The innervation pattern of the radial nerve at the elbow and in the forearm. J. Hand Surg., 23(2):167-9, 1998

Caetano, E. B.; Vieira, L A.; Sabongi-Neto, J. J.; Caetano, M. B. F.; Sabongi, R. G.; Martinez, L.; Ogata, K. K. \& Boni, E. B. S. Anatomical study of pronator teres muscle innervation and clinical significance in nerve transfer. Int. J. Morphol., 36(4):1500-8, 2018.

Chen, G.; Jiang, H.; Liu, A. T.; Zhang, J. L.; Lin, Z. H.; Dang, R. S.; Yu, D. Z.; Li, W. P. \& Liu, B. L. Neurovascular details about forearm muscles: applications in their clinical use in functional muscular transfer. Surg. Radiol. Anat., 32(1):3-8, 2009. 
Fridén, J. \& Gohritz, A. Brachialis-to-extensor carpi radialis longus selective nerve transfer to restore wrist extension in tetraplegia: case report. J. Hand Surg. Am., 37(8):1606-8, 2012.

García-López, A.; Navarro, R.; Martinez, F. \& Rojas, A. Nerve transfers from branches to the flexor carpi radialis and pronator teres to reconstruct the radial nerve. J. Hand Surg. Am., 39(1):50-6, 2014.

Hovelacque, A. Anatomie des Nerfs Crâniens et Rachidiens et du Système Grand Sympathique Chez l'Homme. Paris, G. Doin et cie, 1927. pp.499-501.

Kerver, A. L.; Carati, L.; Eilers, P. H.; Langezaal, A. C.; Kleinrensink, G. J. \& Walbeehm, E. T. An anatomical study of the ECRL and ECRB: feasibility of developing a preoperative test for evaluating the strength of the individual wrist extensors. J. Plast. Reconstr. Aesthet. Surg., 66(4):543-50, 2013.

Konjengbam, M. \& Elangbam, J. Radial nerve in the radial tunnel: anatomic sites of entrapment neuropathy. Clin. Anat., 17(1):21-5, 2004.

Linell apud Hovelacque, A. Anatomie des Nerfs Crâniens et Rachidiens et du Système Grand Sympathique Chez l'Homme. Paris, G. Doin et cie, 1927. pp.499-501.

Liu, J.; Pho, R. W.; Pereira, B. P.; Lau, H. K. \& Kumar, V. P. Distribution of primary motor nerve branches and terminal nerve entry points to the forearm muscles. Anat. Rec., 248(3):456-63, 1997.

Moore, K.; Dailey, A. \& Agur, A. Anatomía con Orientación Clínica. $7^{\text {a }}$ ed. Barcelona, Wolters Kluwer, 2013.

Pet, M. A.; Ray, W. Z.; Yee, A. \& Mackinnon, S. E. Nerve transfer to the triceps after brachial plexus injury: report of four cases. J. Hand Surg. Am., 36(3):398-405, 2011

Safwat, M. D. \& Abdel-Meguid, E. M. Distribution of terminal nerve entry points to the flexor and extensor groups of forearm muscles: an anatomical study. Folia Morphol. (Warsz), 66(2):83-93, 2007.

Segal, R. L.; Wolf, S. L.; DeCamp, M. J.; Chopp, M. T. \& English, A. W. Anatomical partitioning of three multiarticular human muscles. Acta. Anat. (Basel), 142(3):261-6, 1991.

Sunderland, S. Metrical and non-metrical features of the muscular branches of the radial nerve. J. Comp. Neurol., 85:93-111, 1946.

\author{
Dirección para correspondencia: \\ Dr. Enrique Olave \\ Facultad de Medicina \\ Universidad de La Frontera \\ Temuco - CHILE
}

\section{Email: enrique.olave@ufrontera.cl}

Recibido : 11-12-2018

Aceptado: 19-02-2019 